\title{
Early Ventricular Dysfunction in Type II Diabetes: Role of Metabolic Unbalance
}

\author{
Christian Cadeddu ${ }^{1 *}$, Silvio Nocco ${ }^{2}$, Davide Piano ${ }^{2}$, Martino Deidda ${ }^{2}$, Efisio Cossu², Marco Giorgio Baroni ${ }^{2}$ and Guseppe Mercuro ${ }^{2}$
}

${ }^{1}$ Department of Cardiovascular and Neurological Sciences, University of Cagliari, Italy

${ }^{2}$ Department of Internal Medicine, University of Cagliari, Italy

\begin{abstract}
Introduction: Diabetic cardiomyopathy is characterized by early myocardial systolic and diastolic impairment. Many studies have highlighted reduced contractile reserve in patients affected by Metabolic Syndrome (MS) and in the early stages of diabetes mellitus, however, it has not been confirmed by all the authors. A conclusive hypothesis on the basis of this early myocardial dysfunction needs to be ascertained.

Methods: Forty patients: 20 (age $59 \pm 7$ years) with type II diabetes, diagnosed at least 5 years earlier and treated with insulin sensitizers, 20 with MS (WHO definition; age $57 \pm 10$ years), and 20 age- and sex-matched healthy controls were studied. MS patients with elevated insulin resistance (HOMA-IR >2.8), but no arterial hypertension with evidence of cardiac hypertrophy, were selected. In order to exclude ischemic heart disease and to evaluate left ventricle contractile reserve (LVCR), dobutamine echocardiography was performed on all patients.

Results: No significant differences were shown by basal echocardiography between the 3 groups of subjects except for left ventricular mass, which was higher in diabetics than in MS and control subjects $\left(78.8 \pm 17.9 \mathrm{~g} / \mathrm{m}^{2} \mathrm{vs}\right.$. $64.3 \pm 11.1 \mathrm{~g} / \mathrm{m}^{2}$ and $58.2 \pm 9.4 \mathrm{~g} / \mathrm{m}^{2}$, respectively; $p=n s$ and $\left.p<0.05\right)$. A significant decrease in delta longitudinal Strain Rate was noticed in the MS group compared to diabetics and controls $\left(0.59 \pm 0.29 \mathrm{~s}^{-1} \mathrm{vs} .1 .07 \pm 0.44 \mathrm{~s}^{-1}\right.$ and $1.11 \pm 0.43 \mathrm{~s}^{-1}$, respectively; $p<0.001$ )

Conclusion: The present findings show that insulin resistance is responsible for an initial myocardial impairment in patients with MS. This negative effect is not evident in the diabetic patients, probably due to the optimal treatment with insulin sensitizers. In our opinion, insulin resistance is able to modulate heart function, as testified by a depressed myocardial contractility reserve. Our data strengthen the need for the utmost attention to be paid to insulin resistance condition and to the opportunity of early therapeutic intervention.
\end{abstract}

Keywords: Stress echocardiography; Type II diabetes; Insulin resistance

\section{Introduction}

Type II diabetes is frequently associated with heart failure, a common cause of cardiac death in diabetic patients [1]. Hence, an important direction in the management of diabetes is the screening for and treatment of preclinical ventricular abnormalities [2].

In animal models of early stage uncompensated diabetes a reduction in radial and longitudinal left ventricular (LV) function and contractile reserve can be detected with Doppler myocardial imaging before the onset of reduced global LV function [3].

Metabolic Syndrome (MS)does not represent a single morbid entity but rather a clustering of closely correlated risk factors that, by acting together, significantly increase the probability of developing diabetes type II mellitus (DM) and cardiovascular dysfunction $[4,5]$.

Importantly, the increased prevalence of abnormal structure and heart failure is associated with insulin resistance (IR) even when not accompanied by frank DM [6,7]. Patients with nonischemic cardiomyopathy are not only more insulin-resistant than a healthy control population but also more than patients with coronary artery disease [8].

Dobutamine stress echocardiography (DSE) is widely used in patients with coronary artery disease or dilated cardiomyopathy, and allows for evaluation of left ventricular contractile reserve (LVCR] [9].

Fang et al. showed that, while patients with DM had impaired basal myocardial Doppler tissue imaging parameters at rest when compared with controls, the augmentation of myocardial Doppler tissue imaging in response to incremental pharmacological stress with dobutamine was normal [10].

However the assessment of LV longitudinal functional reserve in other studies appeared to be impaired in patients with type 2 diabetes after DSE [11] or exercise echocardiography [12].

More recently, reduced LVCR has been shown in insulin resistant patients following exercise [13] or DSE [14]. We hypothesized that IR is the most important determining factor in the development of impaired LVCR in MS and diabetic patients. For this reason, the response to a stress test with dobutamine of an insulin-resistant MS population was compared to that of a type II diabetes population treated with insulinsensitizers and normal subjects.

*Corresponding author: Christian Cadeddu, Department of Cardiovascular and Neurological Sciences, University Hospital of Cagliari, Strada Statale 554, Km 4.500, 09042 Monserrato (Cagliari), Italy, Tel/Fax: (00 39) 070 675-4955/4991; E-mail: cadedduc@unica.it

Received April 22, 2013; Accepted May 21, 2013; Published May 27, 2013

Citation: Cadeddu C, Nocco S, Piano D, Deidda M, Cossu E, et al. (2013) Early Ventricular Dysfunction in Type II Diabetes: Role of Metabolic Unbalance. J Diabetes Metab S13: 004. doi:10.4172/2155-6156.S13-004

Copyright: (c) 2013 Cadeddu C, et al. This is an open-access article distributed under the terms of the Creative Commons Attribution License, which permits unrestricted use, distribution, and reproduction in any medium, provided the original author and source are credited. 


\section{Method}

Forty high cardiovascular (CV) risk patients, consecutively selected from a population of individuals screened at the Diabetic Centre of our University Hospital, were enrolled. Of these, 20 had type 2 diabetes mellitus and $20 \mathrm{MS}$ according to WHO definition.

All MS patients presented impaired glucose tolerance (IGT) and/ or impaired fasting glucose (IFG) (Table 1), and all were affected by IR, calculated in accordance with the Homeostasis Model Assessment (HOMA) index and defined according to the values of Bonora et al. [15].

All the patients were referred to our stress echo laboratory as they reported a family history of ischemic heart disease. Twenty age, gender and body mass index matched normoglycemic subjects (HOMA $<2.8$; 9 male/11 female), with no structural heart defects or evidence of ischemic heart disease were also enrolled as controls.

Inclusion criteria for all patients were the following: age 20-75 years; carotid intima/media thickness $\geq 1.1$ to define the high $\mathrm{CV}$ risk [16]; an echocardiographic LVEF value $\geq 55 \%$ and absence of echocardiographic wall motion normalities at rest; normal hepatic and renal function (bilirubin $\leq 1.5 \mathrm{mg} / \mathrm{dl}$, creatinine $\leq 2.0 \mathrm{mg} / \mathrm{dl}$ ). Patients were not eligible if they had a history of cardiac disease, hypertension with evidence of cardiac hypertrophy. Additional exclusion criteria were moderate-to-severe valvular heart disease, atrial fibrillation, or severe arrhythmias. Antihypertensive therapy was not an exclusion criterion for diabetic patients, but B-blocker therapy, if any, was withdrawn 2 weeks prior to carrying out the examination to avoid interference with myocardial contractility. Diabetic patients had to have been diagnosed at least 5 years earlier and be under good glycaemic control with insulin sensitizers (i.e. Metformin). The present study was approved by the Ethical Committee of our University Hospital and informed written consent was obtained from all participants.

\begin{tabular}{|c|c|c|c|}
\hline Parameter & MS (N=20) & Diabetes (N=20) & Controls (N=20) \\
\hline Age (yrs) & $57.3 \pm 10$ & $58.9 \pm 7$ & $55.8 \pm 9$ \\
\hline Weight (kg) & $80.7 \pm 11.2^{*}$ & $77 \pm 11$ & $73.6 \pm 9.3$ \\
\hline BMI (kg/m $\left.{ }^{2}\right)$ & $30.8 \pm 5.7^{*}$ & $30 \pm 3^{*}$ & $26.2 \pm 2.8$ \\
\hline Waistcircumference (cm) & $98 \pm 6.2^{*}$ & $96 \pm 7.4$ & $92 \pm 5.3$ \\
\hline Hba1c & $6 \pm 1$ & $7 \pm 1$ & $6 \pm 1$ \\
\hline Fastingglucose (mg/dl) & $114 \pm 13$ & $121 \pm 16^{*}$ & $92 \pm 11$ \\
\hline Homa IR & $8.2 \pm 4.1 \S$ & $1.13 \pm 0.8$ & $1.2 \pm 0.3$ \\
\hline Total cholesterol (mg/dl) & $202 \pm 17$ & $206 \pm 31$ & $192 \pm 29$ \\
\hline HDL (mg/dl) & $52 \pm 10$ & $47 \pm 9$ & $54 \pm 8$ \\
\hline LDL (mg/dl) & $126 \pm 15$ & $134 \pm 26$ & $119 \pm 21$ \\
\hline Triglycerides (mg/dl) & $144 \pm 47$ & $152 \pm 38$ & $118 \pm 30$ \\
\hline Smoking & $20 \%$ & $25 \%$ & None \\
\hline Hypertension (\%) no LVH & $25 \%$ & $30 \%$ & $30 \%$ \\
\hline & Carbohydrates metabolism & \\
\hline NG & $0 \%$ & N/A & N/A \\
\hline IFG & $36 \%$ & N/A & N/A \\
\hline IGT & $63 \%$ & N/A & N/A \\
\hline IGT+IFG & $11 \%$ & N/A & N/A \\
\hline
\end{tabular}

Data are expressed as mean $\pm \mathrm{SD}$

Abbreviations: BMI: Body Mass Index; LVH: Left Ventricle Hypertrophy; NG: Normal Glucose; IFG: Impaired Fasting Glucose; IGT: Impaired Glucose Tolerance; N/A: Notapplicable

$p<0.05$ vs controls; $\S p<0.05$ MS vs diabetes

Table 1: Clinical and laboratory data.

\section{Baseline analysis}

Complete cardiovascular assessment, clinical evaluation, ECG, mono and bi-dimensional Doppler echocardiogram and complete hematochemical tests, as well as the oral glucose tolerance test (baseline glycaemia and after 120 ' following a 75 g glucose load) and insulinemia dosage, were performed in all patients at enrolment.

\section{Conventional echocardiography and Tissue Doppler Imaging (TDI)}

All participants were instructed on the medical environment and instrumentation before testing. Echocardiographic images were recorded using a TDI imaging and raw data acquisition system (Toshiba Aplio; Toshiba Corp., Tochigi, Japan). LVEF was obtained from the apical 4- and 2-chamber views according to Simpson's rule and was considered abnormal when $<55 \%$. Pulsed Wave Doppler (PWD) examination was carried out of the LV inflow from the 4-chamber view with the sample volume placed between the mitral leaflet tips and the early (E) and late (A) diastolic peak velocities; E deceleration time $($ DecT) was measured and E/A ratio was derived.

Longitudinal function was assessed using pulsed TDI at mitral annulus, placing the sample volume in the basal segment of the interventricular septum (IVS.) from the apical 4-chamber view; peak velocities in systole $(\mathrm{Sm})$, early $(\mathrm{Em})$ and late $(\mathrm{Am})$ diastole were measured. LV longitudinal function at baseline and after DSE was evaluated offline from raw data (TDI-Q, Toshiba); longitudinal strain $(\Sigma)$ and strain rate (SR) (average from basal and mid segments of 4 chambers and 2 chamber view) were also quantified based on TDI imaging. All the examinations on each patient were carried out by a single experienced echocardiographer. A simultaneous electrocardiographic trace was also obtained. To reduce inter-observer variability, all echocardiographic data were randomly read by a second experienced observer and an average value for each measurement was calculated. Reproducibility of TDI parameters in our laboratory had been previously documented [17].

\section{DSE}

Dobutamine was administered according to standard 3-min incremental protocol (5 to $40 \mu \mathrm{g} / \mathrm{kg} / \mathrm{min}$ ), and atropine was injected in patients who had not achieved $85 \%$ of their maximal heart rate (1 $\mathrm{mg}$, with increments of $0.25 \mathrm{mg}$ until the target heart rate) [18]. TDI of the 4 and 2 chamber view was recorded at rest and at peak dose. The 12-lead ECG (Mortara instruments, inc.) was monitored throughout, and blood pressure (BP) recorded at rest and at 3-min intervals during infusion and recovery. End points of the stress protocol were completion of the protocols, progressive or severe chest pain, severe ventricular arrhythmias, systolic BP of $240 \mathrm{~mm} \mathrm{Hg}$, diastolic BP of 100 $\mathrm{mm} \mathrm{Hg}$, or severe adverse effects.

Tissue Doppler-derived velocities, SR, and $\Sigma$ were measured off line from raw data. According to the 16-segment model of the 4 tomographic LV views, regional wall motion was scored as normal (1), hypokinetic (2), akinetic (3), and dyskinetic (4), and wall motion score index (WMSI) was derived by two experienced observers blinded to the patient's clinical data.

\section{Statistical analysis}

With regards the anthropometric and clinical characteristics of the two groups, continuous variables were compared with ANOVA, and categorical variables were compared with the Fisher's exact test. Differences in echocardiographic parameters were also evaluated using 
Citation: Cadeddu C, Nocco S, Piano D, Deidda M, Cossu E, et al. (2013) Early Ventricular Dysfunction in Type II Diabetes: Role of Metabolic Unbalance. J Diabetes Metab S13: 004. doi:10.4172/2155-6156.S13-004

Page 3 of 5

ANOVA. A two-tailed value of $\mathrm{p}<0.05$ was considered statistically significant. Data are presented as mean $\pm \mathrm{SD}$.

\section{Results}

\section{Characteristics of the population examined}

Of the recruited subjects, two patients (1 with diabetes and 1 with MS) were excluded from the study as they were positive for ischemia at DSE. They then underwent coronary angiography, which confirmed significant coronary stenosis.

Table 1 summarizes the comparison among the groups of examined subjects, with regard to anthropometrical and laboratory data. Patients with MS showed a higher weight compared to controls $(80.7 \pm 11.2$ $\mathrm{kg}$ vs. $73.6 \pm 9.3 \mathrm{~kg} ; \mathrm{p}<0.05)$ and higher waist circumference $(98 \pm 6.2$ $\mathrm{cm}$ vs. $92 \pm 5.3 \mathrm{~cm} ; \mathrm{p}<0.05)$. Moreover compared to controls, subjects suffering from diabetes mellitus and MS had higher body mass indexes $\left(26.2 \pm 2.8 \mathrm{~kg} / \mathrm{m}^{2}\right.$ vs. $30 \pm 3 \mathrm{~kg} / \mathrm{m}^{2}$ vs. $\left.30.8 \pm 5.7 \mathrm{~kg} / \mathrm{m}^{2} ; \mathrm{p}<0,01\right)$.All diabetic patients under pharmacological treatment showed satisfactory metabolic compensation (Hbalc $7 \pm 3$; HOMA $1.13 \pm 0.8$ ). The value of the insulin-resistance index (calculated with HOMA IR) was significantly higher in subjects with MS compared to the diabetic group under insulin- sensitizers treatment $(8.2 \pm 4.1$ vs. $1.13 \pm 0.8 ; \mathrm{p}<0,001)$ and controls $(8.2 \pm 4.1$ vs. $1.2 \pm 0.3 ; \mathrm{p}<0,001)$.

No differences were observed between the three groups of individuals in terms of arterial hypertension, hyperlipidaemia or smoking habit (Table 1).

\section{Echocardiographic parameters}

Table 2 summarizes the echocardiographic data in the two groups of patients and controls. All collected data proved to be comparable in the three groups, except for left ventricle mass indexed for the body surface (LVM (I)) which, even if within normal range in all patients, resulted significantly higher among diabetics compared to controls (78.8 \pm 17.9 vs. $59.3 \pm 10.1 ; \mathrm{p}<0,05)$, and diastolic function. The conventional evaluation of the diastolic function showed a significant reduction of the E/A ratio in the MS group compared to controls. Moreover, both diabetic and MS patients showed a significant increase of end diastolic pressures at TDI evaluation compared to controls as shown by the E/ Em ratio (9.56 \pm 2.7 vs. respectively $13.2 \pm 2.1$ and $13.3 \pm 3.2$; $\mathrm{p}<0.05)$.

\section{Strain rate imaging}

Global longitudinal $\Sigma$ and SR at rest (Table 3) were comparable in the three groups of patients. All participants underwent DSE (see method), which was generally well tolerated. Minor predictable inconveniences, such as palpitations and systolic hypotension occurred, but in no case did discontinuation of the test result. With regard to the outcome of the pharmacological stress test, diabetic subjects revealed normal increase in contractility under dobutamine compared to controls $\left(2.7 \pm 0.53 \sec ^{-1}\right.$ vs. $\left.2.80 \pm 0.32 \mathrm{sec}^{-1} ; \mathrm{p}=\mathrm{ns}\right)$ while MS patients showed reduced longitudinal SR at the peak of drug administration compared to controls and diabetics $(2.44 \pm 0.22 \mathrm{sec}-1$ vs. $2.80 \pm 0.32 \mathrm{vs} .2 .70 \pm 0.53$ sec- $1, \mathrm{p}<0.0001$, table 3). Moreover the Delta SR between peak and basal values of diabetic patients was comparable to controls $(1.10 \pm$ $0.43 \mathrm{sec}^{-1} \mathrm{vs} .1 .07 \pm 0.44 \mathrm{sec}^{-1}$; $\left.\mathrm{p}=\mathrm{ns}\right)$. On the contrary, subjects with MS showed a reduced increase of SR in comparison with controls $(+35.3 \%$ $\pm 21.4 \%$ vs. $+69.5 \% \pm 39.3 \% ; \mathrm{p}<0.001)$ and diabetic patients $(+35.3 \%$ $\pm 21.4 \%$ vs. $70.2 \% \pm 33.9 \% ; \mathrm{p}<0.001$ ) (Figure 1 ), thereby revealing reduced LVCR. There were no changes in the $\Sigma$ (Table 3), due to the influence exercised on this parameter by stroke volume, which tends to be reduced after dobutamine, for the increase in heart rate and the resulting reduction in diastolic filling.

\section{Discussion}

Our study examined the contractile reserve of subjects with diabetes mellitus diagnosed not less than 5 years earlier in comparison with MS

\begin{tabular}{|c|c|c|c|}
\hline Parameter & MS (N=19) & Diabetes $\mathbf{( N = 1 9 )}$ & Controls $(\mathbf{N}=20)$ \\
\hline EDD $(\mathrm{mm})$ & $49.3 \pm 4.5$ & $52 \pm 5.9$ & $47.6 \pm 5.4$ \\
\hline EDV $(\mathrm{ml})$ & $87.2 \pm 17.7$ & $92.1 \pm 10$ & $74.1 \pm 8.2$ \\
\hline LAA $\left(\mathrm{cm}^{2}\right)$ & $19.21 \pm 4.14$ & $18.91 \pm 3.31$ & $17.6 \pm 2.69$ \\
\hline LVM $(\mathrm{I})\left(\mathrm{g} / \mathrm{m}^{2}\right)$ & $64.3 \pm 11.1$ & $78.8 \pm 17.9 \S^{*}$ & $58.2 \pm 9.4$ \\
\hline LVEF $(\%)$ & $62 \pm 4.1$ & $64 \pm 5.3$ & $63 \pm 3.8$ \\
\hline E/A ratio & $0.89 \pm 0.25^{*}$ & $0.97 \pm 0.21$ & $1.01 \pm 0.25$ \\
\hline DecT $(\mathrm{sec})$ & $0.221 \pm 0.05$ & $0.216 \pm 0.04$ & $0.206 \pm 0.03$ \\
\hline Sm $(\mathrm{cm} / \mathrm{sec})$ & $48.7 \pm 8.1$ & $49.9 \pm 9.5$ & $52.3 \pm 6.5$ \\
\hline Em $(\mathrm{cm} / \mathrm{sec})$ & $56.5 \pm 7.7$ & $59.9 \pm 9.2$ & $68.3 \pm 8.9$ \\
\hline E/Em & $13.2 \pm 2.1^{*}$ & $13.3 \pm 3.2^{*}$ & $9.56 \pm 2.7$ \\
\hline
\end{tabular}

Data are expressed as mean $\pm \mathrm{SD}$

Abbreviations: EDD: End Diastolicdiameter; EDV: End Diastolic Volume; LAA Leftatrium Area; LVM: Leftventricular Mass; LVEF: Left Ventricular Ejection Fraction E/A: Early and Late Diastolic peak Velocity Ratio; DecT E: Wavedeceleration time S TD: Systolicpeakvelocity; E TD: Earlydiastolicpeakvelocity; E: Eearly PWD; TD: Diastolicpeakvelocity ratio. ${ }^{*} p<0.05$ vs controls $\S \mathrm{p}<0.05 \mathrm{MS}$ vs diabetes

Table 2: Transthoracic conventional echocardiographic and TDI parameters.

\begin{tabular}{|c|c|c|c|}
\hline & MS (N=19) & Diabetes ( $N=19)$ & Controls $(\mathrm{N}=\mathbf{2 0})$ \\
\hline \multicolumn{4}{|c|}{ Strain Rate imaging } \\
\hline \multicolumn{4}{|c|}{ Basal } \\
\hline$\Sigma(\%)$ & $21.2 \pm 6.27$ & $22.4 \pm 8.1$ & $23.1 \pm 5.4$ \\
\hline $\operatorname{SR}\left(\sec ^{-1}\right)$ & $1.84 \pm 0.30$ & $1.63 \pm 0.42$ & $1.70 \pm 0.26$ \\
\hline \multicolumn{4}{|c|}{ High dose dobutamine } \\
\hline$\Sigma(\%)$ & $22.9 \pm 4.13$ & $23.5 \pm 6.6$ & $25.5 \pm 3.17$ \\
\hline $\operatorname{SR}\left(\sec ^{-1}\right)$ & $2.44 \pm 0.22^{*}$ & $2.70 \pm 0.53^{*}$ & $2.80 \pm 0.32^{*}$ \\
\hline \multicolumn{4}{|c|}{$\Delta$ Stress } \\
\hline$\Sigma(\%)$ & $+1.7 \pm 2.1(+8 \%)$ & $+1.1 \pm 1.3(+5 \%)$ & $+2.4 \pm 2.2(+10 \%)$ \\
\hline $\operatorname{SR}\left(\sec ^{-1}\right)$ & $0.59 \pm 0.29(+35 \%)^{*}$ & $1.07 \pm 0.44(+70 \%)^{*}$ & $1.11 \pm 0.43(+69 \%)^{*}$ \\
\hline
\end{tabular}

Data are expressed as mean $\pm S D$

Abbreviations: SR: Strain Rate; $\Sigma$ Strain. ${ }^{*} \mathrm{P}<0.001$ vs MS

Table 3: Longitudinal $\Sigma(\%)$ and SR (s-1).

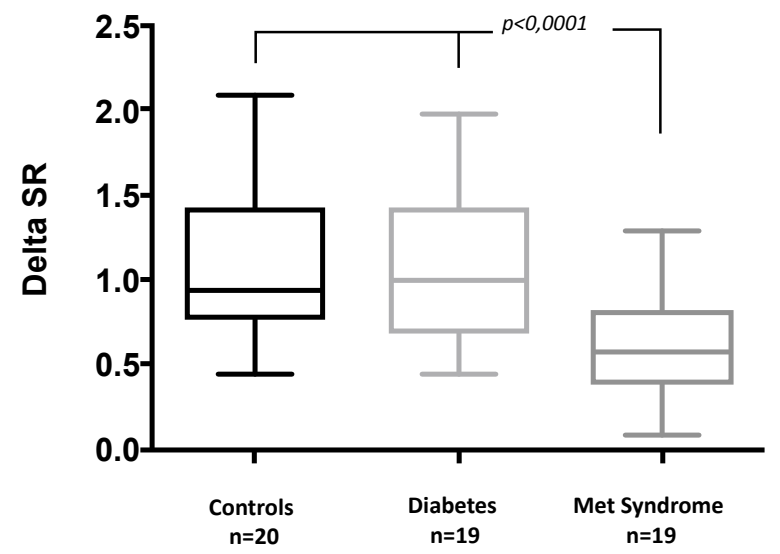

Figure 1: Delta SR absolute values in the three groups. Data is expressed as mean value \pm SD 
and a control group. Results showed: a. a considerably higher value of insulin-resistance in patients with MS compared to diabetic subjects treated with insulin-sensitizers; b. impaired diastolic function both in MS and diabetic patients; c. longitudinal $\Sigma$ and SR values statistically comparable in the two groups and between them and healthy controls; d. a reduced increase in longitudinal SR values at peak stress in MS patients compared to diabetic and control subjects.

No evidence of Strain modifications was witnessed confirming the close connection between Strain and stroke volume, which tends to reduce when the dobutamine dose is increased (because of the increase in heart rate and the consequent reduction in diastolic filling) [19]. It is now certain that diabetes mellitus can cause systolic and diastolic dysfunction in patients suffering from this disease [20].Traditionally, when determining the contractile dysfunction caused by diabetes, the mediation of an accelerated atherogenesis and/or hypertension was considered necessary. More recently, a more controversial hypothesis has been developed according to which, diabetes harms the cardiac structure and its function independently from blood pressure and coronary disease [21]. At present, the connections between the metabolic disorders related with diabetes and the cell effects up to the appearance of clinical non-ischemic manifestation are particularly stressed.

Perfusion abnormalities are likely a component of diabetic cardiomyopathy, but significant contributions also derive from disturbed myocardial energetics, fibrosis, and autonomic dysfunction. These changes correlate with impairment of functional capacity and lack of myocardial reserve may explain the propensity of patients with diabetes mellitus to develop heart failure after myocardial injury [22]. The initiating myocardial insult is clouded by several potential interacting mechanisms, and the etiology is likely multifactorial.

As in our study, a previous work has also shown that myocardial velocity response to dobutamine stress is preserved in DM, which would not be expected with a vascular etiology, [10] in which the lack of blood flow reserve would be expected to compromise the stress response. Moreover, a study of contrast echocardiography and myocardial deformation showed both myocardial perfusion and function to be abnormal in long-termdiabetic subjects (average disease time 10 years), but lacking any significant correlation [23]. More recently a reduced stress response of the diabetic myocardium with strain rate imaging has been evidenced [11]. Although the results are not completely conclusive since patients with LV hypertrophy, which may itself reduce LV longitudinal function, had been enrolled.

Epidemiological evidence suggests more than simply a correlation between IR and heart failure, demonstrating that IR precedes heart failure rather than occurring as a consequence of it. IR has been shown to be able to predict the subsequent development of heart failure in a Swedish population, independently of all established risk factors, including diabetes mellitus itself [24].

The cardiovascular mortality rate in IGT is twice as much as that of normal glucose tolerance, 2 to 3 fold higher in type II diabetes mellitus [25]. Because high mortality cannot be fully explained by conventional cardiovascular risk factors, it has been attributed to factors related to IR and its potentially adverse effects on myocardial metabolism and endothelial function [26].

In our study the major difference of the 3 groups is the result of the presence of IR. At myocardial level insulin causes inhibition of apoptosis, hypertrophy/fibrosis stimulus and NO production [27]. Moreover a change in cardiac metabolism can be associated with a condition of insulin-resistance, in the absence of other causative factors. Several studies suggest an alteration in myocardial energetic metabolism caused by IR that could lead to impairment of myocardial ATP production [28-30]. This mild lack in ATP availability could become significant in conditions of major energetic demand, as during catecholaminergic stress. The reduced LVRC evidenced only in the MS group suggests a major role of insulin resistance in determining myocite dysfunction independently of diabetes itself.

To our knowledge, this study is the first to compare two populations of patients with glycaemic dysmetabolism, that can be distinguished for insulin-resistance, present in subjects with MS and virtually corrected in those with diabetes mellitus. We showed a significantly reduced SR increase during dobutamine, a parameter that is sensitive to the slightest changes in the inotropic state of the heart. Vasculopathy stands as a "usual suspect" in the complications of diabetes, and a preserved myocardial velocity response to dobutamine stress would not be expected with a vascular etiology.

The evidence of a marked reduction in LVCR only in the subjects with MS that we examined could be explained by the mentioned "metabolic hypothesis". At the same time, the absence of a similar change in the group of diabetic patients could be connected to the use of insulin-sensitizing molecules, able to increase its supply.

The functional changes we observed appear to be an IR-related cardiomiopathy that differs from the diabetic form. In fact, the macroscopic morphological changes of myocardium, such as the wellknown hypertrophy caused by hyperinsulinemic or hyperadrenergic stimulus were not observed. These findings are probably related to the early stage of metabolic dysfunction in the MS group.

In conclusion, our data confirm that MS patients show an early reduction in myocardial longitudinal contractile reserve. This dysfunction, not detected in the diabetic subjects with a good pharmacologic metabolic compensation, could be related to IR state.

Our data seem to suggest a higher attention to be paid to IR state and for potential early therapeutic intervention, able to improve cardiopulmonary and endothelial function [31], especially in MS patients with high HOMA levels [32].

\section{Acknowledgments}

The authors thank Mr. Barry Mark Wheaton for the final linguistic revision of the manuscript.

\section{References}

1. Tang WH, Young JB (2001) Cardiomyopathy and heart failure in diabetes Endocrinol Metab Clin North Am 30: 1031-1046.

2. Struthers AD, Morris AD (2002) Screening for and treating left-ventricula abnormalities in diabetes mellitus: a new way of reducing cardiac deaths. Lancet 359: 1430-1432.

3. Weytjens C, Cosyns B, D'hooge J, Droogmans S, Lahoutte T, et al. (2010) Evaluation of contractile function and inotropic reserve with tissue velocity strain and strain rate imaging in streptozotocin-induced diabetes. Eur $\mathrm{J}$ Echocardiogr 11: 622-629.

4. Lakka HM, Laaksonen DE, Lakka TA, Niskanen LK, Kumpusalo E, et al. (2002) The metabolic syndrome and total and cardiovascular disease mortality in middle-aged men. JAMA 288: 2709-2716.

5. Gami AS, Witt BJ, Howard DE, Erwin PJ, Gami LA, et al. (2007) Metabolic syndrome and risk of incident cardiovascular events and death: a systematic review and meta-analysis of longitudinal studies. J Am Coll Cardiol 49: 403 414

6. Iribarren C, Karter AJ, Go AS, Ferrara A, Liu JY, et al. (2001) Glycemic control and heart failure among adult patients with diabetes. Circulation 103: 26682673. 
Citation: Cadeddu C, Nocco S, Piano D, Deidda M, Cossu E, et al. (2013) Early Ventricular Dysfunction in Type II Diabetes: Role of Metabolic Unbalance. J Diabetes Metab S13: 004. doi:10.4172/2155-6156.S13-004

Page 5 of 5

7. Ilercil A, Devereux RB, Roman MJ, Paranicas M, O'Grady MJ, et al. (2002) Associations of insulin levels with left ventricular structure and function in American Indians: the strong heart study. Diabetes 51: 1543-1547.

8. Fang ZY, Prins JB, Marwick TH (2004) Diabetic cardiomyopathy: evidence, mechanisms, and therapeutic implications. Endocr Rev 25: 543-567.

9. Naqvi TZ, Goel RK, Forrester JS, Siegel RJ (1999) Myocardial contractile reserve on dobutamine echocardiography predicts late spontaneous improvement in cardiac function in patients with recent onset idiopathic dilated cardiomyopathy. J Am Coll Cardiol 34: 1537-1544.

10. Fang ZY, Najos-Valencia O, Leano R, Marwick TH (2003) Patients with early diabetic heart disease demonstrate a normal myocardial response to dobutamine. J Am Coll Cardiol 42: 446-453

11. Galderisi M, de Simone G, Innelli P, Turco A, Turco S, et al. (2007) Impaired inotropic response in type 2 diabetes mellitus: a strain rate imaging study. Am J Hypertens 20: 548-555.

12. Ha JW, Lee HC, Kang ES, Ahn CM, Kim JM, et al. (2007) Abnormal left ventricular longitudinal functional reserve in patients with diabetes mellitus: implication for detecting subclinical myocardial dysfunction using exercise tissue Doppler echocardiography. Heart 93: 1571-1576.

13. Ha TH, Seo HS, Choo WJ, Choi J, Suh J, et al. (2011) The Effect of Metabolic Syndrome on Myocardial Contractile Reserve during Exercise in Non-Diabetic Hypertensive Subjects. J Cardiovasc Ultrasound 19: 176-182.

14. Cadeddu C, Nocco S, Piano D, Deidda M, Cossu E, et al. (2013) Early impairment of contractility reserve in patients with insulin resistance in comparison with healthy subjects. Cardiovasc Diabetol 12: 66.

15. Bonora E, Kiechl S, Willeit J, Oberhollenzer F, Egger G, et al. (1998) Prevalence of insulin resistance in metabolic disorders: the Bruneck Study. Diabetes 47: 1643-1649.

16. O'Leary DH, Polak JF, Kronmal RA, Manolio TA, Burke GL, et al. (1999) Carotid-artery intima and media thickness as a risk factor for myocardial infarction and stroke in older adults. Cardiovascular Health Study Collaborative Research Group. N Engl J Med 340: 14-22.

17. Cadeddu C, Piras A, Mantovani G, Deidda M, Dessì M, et al. (2010) Protective effects of the angiotensin II receptor blocker telmisartan on epirubicin-induced inflammation, oxidative stress, and early ventricular impairment. Am Heart J 160: 487.e1-487.e7

18. McNeill AJ, Fioretti PM, el-Said SM, Salustri A, Forster T, et al. (1992) Enhanced sensitivity for detection of coronary artery disease by addition of atropine to dobutamine stress echocardiography. Am J Cardiol 70: 41-46.

19. Kubota T, McTiernan CF, Frye CS, Slawson SE, Lemster BH, et al. (1997) Dilated cardiomyopathy in transgenic mice with cardiac-specific overexpression of tumor necrosis factor-alpha. Circ Res 81: 627-635.

20. Hamby RI, Zoneraich S, Sherman L (1974) Diabetic cardiomyopathy. JAMA 229: $1749-1754$.

21. Bertoni AG, Tsai A, Kasper EK, Brancati FL (2003) Diabetes and idiopathic cardiomyopathy: a nationwide case-control study. Diabetes Care 26: 2791 2795

22. Bell DS (2003) Heart failure: the frequent, forgotten, and often fatal complication of diabetes. Diabetes Care 26: 2433-2441.

23. Moir S, Hanekom L, Fang ZY, Haluska B, Wong C, et al. (2006) Relationship between myocardial perfusion and dysfunction in diabetic cardiomyopathy: a study of quantitative contrast echocardiography and strain rate imaging. Heart 92: 1414-1419.

24. Ingelsson E, Sundström J, Arnlöv J, Zethelius B, Lind L (2005) Insulin resistance and risk of congestive heart failure. JAMA 294: 334-341.

25. Haffner SM (2000) Coronary heart disease in patients with diabetes. N Engl J Med 342: 1040-1042.

26. Witteles RM, Fowler MB (2008) Insulin-resistant cardiomyopathy clinica evidence, mechanisms, and treatment options. J Am Coll Cardiol 51: 93-102.

27. Shah A, Shannon RP (2003) Insulin resistance in dilated cardiomyopathy. Rev Cardiovasc Med 4 Suppl 6: S50-57.

28. Boudina S, Sena S, O'Neill BT, Tathireddy P, Young ME, et al. (2005) Reduced mitochondrial oxidative capacity and increased mitochondrial uncoupling impair myocardial energetics in obesity. Circulation 112: 2686-2695

29. Murray AJ, Anderson RE, Watson GC, Radda GK, Clarke K (2004) Uncoupling proteins in human heart. Lancet 364: 1786-1788.

30. Opie LH (2004) The metabolic vicious cycle in heart failure. Lancet 364: 17331734.

31. Cadeddu C, Nocco S, Deidda M, Cadeddu F, Bina A, et al. (2012 Cardiopulmonary and endothelial effects of metformin treatment in an insulin resistant population. Int J Cardiol 158: 302-304.

32. Cadeddu C, Nocco S, Deidda M, Cadeddu F, Bina A, et al. (2013) Relationship between high values of HOMA-IR and cardiovascular response to metformin Int J Cardiol 167: 282.
This article was originally published in a special issue, Type 2 Diabetes Mellitus- Disease, Diagnosis \& Treatment handled by Editors. Dr. Judit Bene, University of Pécs, Hungary; Eun Seok Kang, Yonsei University College of Medicine, Korea 